\title{
Haine de la «musique » = peur du non-sens : trouées et censures de la vocalité poétique
}

Jean-Pierre Bobillot

\section{OpenEdition}

\section{Journals}

Édition électronique

URL : http://journals.openedition.org/recherchestravaux/453

DOI : 10.4000/recherchestravaux.453

ISSN : 1969-6434

Éditeur

UGA Éditions/Université Grenoble Alpes

\section{Édition imprimée}

Date de publication : 15 mai 2011

Pagination : 119-134

ISBN : 978-2-84310-200-4

ISSN : 0151-1874

Référence électronique

Jean-Pierre Bobillot, « Haine de la « musique » = peur du non-sens : trouées et censures de la vocalité poétique », Recherches \& Travaux [En ligne], 78| 2011, mis en ligne le 15 novembre 2012, consulté le 08 septembre 2020. URL : http://journals.openedition.org/recherchestravaux/453 ; DOI : https://doi.org/ 10.4000/recherchestravaux.453 
Jean-Pierre BobILlot

\section{Haine de la «musique» = peur du non-sens : trouées et censures de la vocalité poétique}

«la vocation des mots c'est leur vocalise même»

Michel Seuphor

\section{Du jubilus au «bruit»}

On connaît la chanson : il irait de soi que Le Poëme a toujours été voix, est Voix - ou quasi indifféremment, Musique «... avant toute chose ${ }^{\mathrm{I}}$ ! Imaginaire «bande son» doublant toute lecture silencieuse : voix de l'auteur ou du texte, $\mathrm{du}$ "narrateur", du "poète», - voix du Verbe ou musique des Sphères, - musique mentale, des mots, de la langue, des vers, de sa langue à lui! À la manière de quelque ineffable essence...

Mais ceux qui ressassent cela, ce n'est pas pour parler de poètes-proférateurs comme l'Artaud de Pour en finir avec le jugement de dieu - ni plus largement, à propos de «Poésie sonore» ou "action"

Car, ils ignorent ces voix effectivement proférées, voire enregistrées - restituées telles quelles ou transformées, triturées, ou diversement orchestrées -, au profit d'acceptions concurrentes, toutes également analogiques, de ce concept de "voix» - ou de «musique» : écran, autant que fourre-tout, se ramenant à telle convergence de marques énonciatives, ou stylistiques, à quelque empirique notation de «l'oral dans l'écrit»...

I. Sur «la musique comme imaginaire de la littérature» et, en particulier, de la poésie, voir Fr. Escal, Contrepoints. Musique et littérature, Klincksieck, I990, en particulier p. IIO-I4I; sur l'«idéologie musicale» de la poésie aux temps symbolistes et postsymbolistes, voir L. Jenny, La fin de l'intériorité, Presses universitaires de France, 2002, p. 47-69.

2. J.-P. Bobillot, Poésie sonore. Éléments de typologie historique, Le Clou dans le fer, 2009, notamment p. 26-32. 
André Spire déjà, n’ironisait-il pas :

De la musique! de la musique, de la musique avant toute chose! Soit! si cela veut dire avec Carlyle : «Allez assez en avant, il y a de la musique partout.»

Mais de quelle musique s'agit-il dans ce délire de comparaisons? De la musique antique; de la musique moderne; de la musique orientale, extrême-orientale ou européenne? De la monodie, de la polyphonie [...], de la musique des sphères, ou tout simplement de la musique du rossignol ${ }^{3}$ ?

Or cette attitude n'est pas sans précédents : ceux qui, en poésie, ne veulent «entendre» que ces prétendues voix - cette prétendue musique -, aussi muettes qu'inaudibles, mais refusent de prêter l'oreille ou la moindre "valeur poétique» à celle du poète lisant, et a fortiori aux voix diversifiées (et assumées) de la "sonore», jouent un rôle similaire à celui des ennemis du chant et de la musique dans la liturgie chrétienne, qui s'opposèrent durant des siècles (quelquefois, par la violence) aux progrès de sa vocalisation et de son instrumentation - attribuées aux menées sournoises du Malin ${ }^{4}$ : celui qui, susceptible de revêtir toutes les formes, au bout du compte, n'en a aucune...

Se fondant sur une interprétation restrictive de la formule de saint Paul : in cordibus vestris, ils en vinrent à promouvoir une "conception minimaliste» suivant laquelle le chant ne pouvait être qu' "intérieur» - aussi muet qu' inaudible, sinon pour Dieu (Poizat, p. 4I-43). Parallèlement, si la méfiance (et quelquefois la haine) a toujours été grande, chez les législateurs, quant à l'usage jugé excessif des instruments dans le culte (ainsi Érasme fustigeait-il les «braillements tumultueux de voix diversifiées», ou le "hennissement de l'orgue» qu'on entendait dans les églises), il en va tout autrement de la référence allégorique à ces instruments, qui resta toujours «une constante du discours apologétique», comme le souligne Michel Poizat citant saint Augustin :

Vous êtes la trompette, le psaltérion, la cithare, le tympanon, le chœur, les cordes et l'orgue, et les cymbales de la jubilation bien sonnantes, parce que consonantes. Vous êtes tout ceci; ici rien de vil, rien de fugitif, rien de matériel ne doit nous venir en pensées. (Ibid., p. I30.)

Bref, qu'il s'agisse de l'histoire du chant et de la musique sacrés dans la tradition chrétienne, ou de la question de la voix dans la poésie moderne et contemporaine, de fortes résistances surgissent "chaque fois qu'une avancée nouvelle tend à émanciper la dimension purement vocale» - c'est-à-dire «matérielle»-, aux dépens de la domination du signifié - censément immatériel (Verbe, Parole, Idée). Et dans les deux cas, c'est une égale répression de la jouissance vocale, assimilée au triomphe de l'informe sur la Forme: de

3. Plaisir poétique et plaisir musculaire [1949], Corti, I986, p. 6-7.

4. M. Poizat, La Voix du diable, Métailié, I99I, notamment p. 20-50.

5. Je souligne. 
l'informulable (la vocalité) sur le formulé (le Verbe). Variantes atténuées d'une même castration : dans les deux cas, c'est bien "une acception quasiment métaphorique - et idéaliste - du chant [voix ou musique] qu'il convient de développer ${ }^{6}$ (Poizat, p. 40).

Si d'aucuns en ignorent tout, certains, les ayant entendues, refusent délibérément de prendre en compte ces poésies ou de leur accorder la moindre «valeur poétique». Ainsi, Jean Cohen :

Le lettrisme s'est voulu poème. Par là, il s'est condamné lui-même. Un poème qui ne signifie pas n'est plus poème, parce qu'il n'est plus langage.

Tzvetan Todorov :

La poésie phonique n'est intéressante qu'en tant qu'elle constitue une limite de la poésie (du langage); on doit pouvoir l'atteindre mais non la pratiquer de manière indépendante : elle s'intégrerait alors à la musique ou à la peinture; mais c'est une pauvre musique et une peinture bien limitée.

François Rigolot :

Les divers bruitages du dadaïsme ou du lettrisme peuvent avoir un intérêt historique ou anthropologique certain; leur pouvoir d'incantation peut même être très grand et provoquer des réactions émotives intenses chez le lecteur. Cependant ils restent en deçà du seuil poétique proprement dit parce que le langage qu'ils utilisent n'a pas de valeur communicative identifiable?.

Cette dernière phrase atteste la forte composante théologique qui caractérise la position de ces néopuritains. Quel est en effet l'argument majeur des ennemis du chant et de la musique sacrés? C'est que toute l'énergie physique et mentale et toute l'attention apportées à la vocalité ou à l'instrumentation (et au plaisir qui s'y attache) sont par là même soustraites à ce qui doit être l'unique objet de la liturgie : la présentation exacte - formelle, à la syllabe près - de la parole divine aux fidèles assemblés, la "préservation totale de la lettre du texte et de son intelligibilité dans la clarté la plus complète de son élocution", ou la proclamation, par la communauté des fidèles à l'unisson, d'une louange ou d'une prière adressée à Dieu.

On est donc bien dans "une logique de la communication ${ }^{\mathrm{I}}{ }^{\text {» (Poizat, }}$ p. 2I-25), suivant laquelle tout ce qui porte atteinte à l'intégrité du message

6. Il souligne.

7. Structure du langage poétique, Flammarion, coll. "Champs», 1966, p. 31. À le vrai dire, Isou et Lemaître avaient anticipé - et ruiné d'avance - l'argument, en affirmant que la lettrie n'était pas plus poésie que musique, mais qu'elle instaurait un tout autre champ, et par làmême les dépassait, et les périmait...

8. "Le sens des sons", Seuil, Poétique, n II, I972, p. 458.

9. "Le poétique et l'analogique», Sémantique de la poésie, Seuil, coll. "Points», I979, p. I66-I67 (il souligne).

Io. Je souligne, infra. 
- soit, de l'information - doit être considéré comme bruit - c'est-à-dire, comme informe - et éliminé : que le message en question soit la parole divine, celle de la communauté des fidèles... ou celle du poète :

Doit être impitoyablement rejeté tout ce qui s'attaque à la parole divine, que ce soit au moyen d'un procédé vocal qui ne s'en tient plus à sa place d'humble servante de cette parole ou que ce soit par le recours à d'autres paroles que celles qui sont considérées comme "authentiques».

Soit : "Doit être impitoyablement rejeté tout ce qui s'attaque au message véhiculé par le poème, que ce soit au moyen de procédés mettant en avant la voix proférée ou enregistrée, ou que ce soit par le recours à d'autres types de textes - relevant du phonétisme, des mots inventés ou recourant aux formes de la langue parlée ordinaire - que ceux qui sont généralement considérés comme relevant du prétendu "langage poétique"."

$\mathrm{Ou}$ : la loi du Verbe, à laquelle répond la place centrale accordée au «message» dans le schéma de la communication (Shannon) comme dans celui des composantes et fonctions du langage (Jakobson), fondait une logique communicationnelle visant à marginaliser tout ce qui risque de venir déborder, obscurcir ledit message, idéalement dématérialisé, décontextualisé - pure forme -, désincarné. Alors que, dans la cantillation puis la psalmodie des premiers siècles, chaque unité de temps mélodique (chaque note) se superposait à une syllabe - préservant ainsi l'intégrité de la Parole divine -, les mélismes qui y apparurent, dans la ferveur de l'Alleluia, y introduisirent des séquences mélodiques (des séries de notes) quelquefois assez longues, chantées «sur une même syllabe du mot et selon des écarts très supérieurs à ceux qui prévalent dans la psalmodie» (Poizat, p. 34) - faisant insidieusement basculer le chant communautaire, de ce vecteur idéal (angélique) de pure et spirituelle signification, qu'il était tenu d'être, à cet espace matériel (satanique) d'impure et charnelle vocalité - informe et insensé - qu'il risquait, à tout instant, de devenir.

Cette transgression - significativement baptisée jubilus - fut d'abord limitée à l'alleluia, terme exclamatif dépourvu de toute signification - information $e t$ forme - autre que de traduire cet état de jubilation (voire de le susciter ou de l'amplifier, de le propager parmi l'assistance); mais le ver était dans le fruit, et toute la chrétienté y mordit à belles dents...

Car, le christianisme n'en est pas moins une pensée de l'acte - et, en premier lieu, de l'incarnation. D'où, chez saint Augustin, ces propos un peu moins austères - et plus nuancés :

Il me semble quelquefois que je prête à ces chants plus qu'il ne le faudrait, en reconnaissant que les paroles saintes elles-mêmes émeuvent nos esprits et les 
enflamment de piété avec une ardeur et une religiosité plus grandes, lorsqu'elles sont ainsi chantées, que si elles ne l'étaient pas - et que les affections de notre âme, dans toute leur diversité, trouvent dans la vocalité du chant les voies appropriées de je ne sais quelle affinité secrète, qui les stimule.

Mais cette délectation de ma chair, à laquelle je ne devrais pas laisser succomber mon esprit, me trompe chaque fois que la sensibilité, au seul prétexte que la raison lui a accordé l'avantage de l'accompagner, ne se résigne pas à demeurer en arrière, et va jusqu'à prétendre passer devant et mener le train! Et voilà comment je tombe dans ce péché, sans m'en rendre compte; après coup pourtant, je m’en rends bien compte...

D'autres fois, redoutant plus que de raison de m'y laisser prendre, je tombe dans un excès de sévérité; au point que, par moments, je voudrais débarrasser mes oreilles - et celles de l'Église tout entière - de toutes ces mélodies, dignes des plus suaves cantilènes, dont on a coutume d'accompagner les Psaumes de David.

[...] Cependant, lorsque [...] je suis ému, non par le chant lui-même, mais par les choses que l'on chante, quand c'est d'une voix limpide et harmonieuse, - alors, je reconnais à nouveau la grande utilité de cette institution.

Ainsi je flotte incertain, entre ce que le plaisir a de périlleux et ce qu'il peut apporter de salutaire; et je suis plutôt conduit [...] à approuver l'usage du chant dans l'Église, de sorte qu'entraîné par les délices de l'oreille, l'esprit encore trop faible puisse s'élever jusqu'au sentiment de la piété. Toutefois, quand il marrive d'être plus ému par le chant que par ce que l'on chante, je confesse que c'est là un péché qui réclame pénitence; et je préférerais alors ne pas entendre chanter ${ }^{\mathrm{II}}$.

Et de même que la notion de «signifiance généralisée ${ }^{\mathrm{I2}}$ » n’a aucunement pour corollaire obligéle «rien de matériel» d'Augustin, ni le "pas de sons dans le langage» de Meschonnic - sauf à idéaliser à son tour ladite signifiance, en l'exonérant de toute matérialité -, de même celui-ci, pourfendeur de Saussure et du saussurisme, se fait-il plus saussurien encore que l'auteur du Cours de linguistique générale lorsqu'il tranche qu' «il n'y a pas de sons dans le langage» (et en déduit qu’il n'y a pas de "poésie sonore»). Car, si le signifiant n’est en lui-même ni phonique ni graphique, il n'est pas de signifiant sans inscription phonique et/ou graphique du signifiant - ce qui suffit à légitimer "poésie visuelle» et "sonore», et toute pratique, disons-la concrète, mettant en œuvre le signifiant.

Censure, dans tous les cas : qui est parvenue à mettre hors jeu - hors Histoire, hors pensée - un siècle au moins de pratiques et de réflexions touchant à la voix et aux techniques d'enregistrement, de restitution et de transformation du son, dans leurs rapports avec la composition poétique et la conception même de la poésie et, plus généralement, du langage.

Un siècle de ce qui rétrospectivement apparaît, de façon manifeste, comme un devenir-sonore du poème.

II. Confessions X, XXXIII, 49-50 (je retraduis).

I2. H. Meschonnic, Critique du Rythme, Verdier, Lagrasse, I982, p. 635. 


\section{«Poésie bruyante»}

J'ai déjà soulignér l'importance historique - décisive - de l'enregistrement de poèmes, par leurs auteurs eux-mêmes, au phonographe, qui eut lieu en décembre I9I3, aux Archives de la Parole, à la Sorbonne, et plus encore peutêtre, de leur diffusion publique, également en Sorbonne, le 27 mai I9I4 - et des conséquences qu’Apollinaire, quasi immédiatement, en tira, quant aux développements et mutations à venir de la poésie, tout au long du siècle.

Pourquoi cet événement n'apparaît-il jamais dans les manuels et les traités; pourquoi ces articles, ces propos de l'auteur d'Alcools et de Calligrammes, dispersés au fil de ses "œuvres complètes" (de I9I3 à 1917), ne sont-ils jamais cités, ni commentés? Qu'y a-t-il de si choquant, dans cette proclamation :

Avant peu, les poètes pourront, au moyen des disques, lancer à travers le monde de véritables poèmes symphoniques. Grâces en soient rendues à l'inventeur du phonographe, Charles Cros, qui aura ainsi fourni au monde un moyen d'expression plus puissant, plus direct que la voix d'un homme imitée par l'écriture ou la typographie ${ }^{14}$.

Et, s'agissant du «livre de poésie», dans celle-ci :

Il est à son déclin. Avant un ou deux siècles, il mourra. Il aura son successeur, son seul successeur possible dans le disque de phonographe et le film cinématographique $^{15}$.

Pourquoi ne pas admettre que c'est là, effectivement, ce qui est arrivé (avec l'«audio-poésie»), et ce qui est en train d'arriver (avec la "vidéo-poésie») ? De quelle autre histoire de la poésie au $\mathrm{Xx}^{\mathrm{e}}$ siècle (et au XXI ${ }^{\mathrm{e}}$ ) a-t-on peur? De quelle autre histoire des avant-gardes se garde-t-on?

Et pourquoi, à propos des «glossolalies» d'Artaud, Évelyne Grossman croyait-elle bon d'affirmer inopinément :

Artaud ne s'est pas fait faute de critiquer le futurisme et son «kaléidoscope frénétique, inutile, pétillant et égaré». De leur côté, les recherches dadaïstes, et singulièrement celles de Ball sur le "poème simultané» [?] ou la poésie sonore qui mettent l'accent sur les valeurs de la voix et le primitivisme magique et religieux, sont très éloignées d'Artaud. Il n'est que de parcourir le fameux poème "Karawane» de I9I7 [sic] que Ball lut au cabaret Voltaire pour distinguer ces suites phoniques fondées sur des écholalies et des symétries acoustiques, des glossolalies écrites d'Artaud ${ }^{16}$.

I3. "La Voix réinventée. Les poètes dans la technosphère : d'Apollinaire à Bernard Heidsieck", Histoires Littéraires n ${ }^{\circ}$ 28, Paris/Tusson, 2006, p. 25 et suiv.

I4. «Nos amis les futuristes» [Is février i9I4] (je souligne).

I5. «M. Guillaume Apollinaire et la nouvelle école littéraire» [24 juin 1917], op. cit., p. 97I, 989. (Et d'ajouter, cum grano salis : "On n'aura plus besoin d'apprendre à lire et à écrire.»)

16. Artaud/Joyce : le corps et le texte, Nathan, I996, p. I85-186. 
Qu'Artaud ne soit pas Marinetti, ni Ball, ni Hausmann, ou Tzara; que diverses soient les voies par lesquelles la voix effectivement proférée en vient à subvertir les formes reçues de la poésie : c'est une évidence. Cependant, cette diversité n'empêche pas que les uns et les autres participent, suivant des modalités quelquefois fort divergentes, de ce même devenir-sonore : d'une même histoire qui est celle, précisément, de l'irruption de la voix propre du poète, comme composante à part entière du poème.

Voix, non seulement, proférée (voire enregistrée), mais, non socialisée - traitresse à la fonction, qui lui est unanimement attribuée, de vecteur d'une bonne communication :

Ô bouches l'homme est à la recherche d'un nouveau langage

Auquel le grammairien d'aucune langue n'aura rien à dire

$[\ldots]$

Mais entêtons-nous à parler

Remuons la langue

Lançons des postillons

On veut de nouveaux sons de nouveaux sons de nouveaux sons

On veut des consonnes sans voyelles

Des consonnes qui pèsent sourdement

Imitez le son de la toupie

Laissez pétiller un son nasal et continu

Faites claquer votre langue

Servez-vous du bruit sourd de celui qui mange sans civilité

Le raclement aspiré du crachement ferait aussi une belle consonne

Les divers pets labiaux rendraient aussi vos discours claironnants

Habituez-vous à roter à volontét

etc. Éloge, donc, du bruit dans la langue - voire, de la langue elle-même comme bruit-, au double sens : communicationnel et esthétique, de bruit : "parasite», et "son laid» ou "sale ${ }^{18}$ ". Voire, à l'inverse, du bruit comme langue ou du moins, comme lettres, si l'on rapproche ces vers subséquents d'Apollinaire ${ }^{19}$ :

Parlez avec les mains faites claquer vos doigts

Tapez-vous sur la joue comme sur un tambour

$$
\text { Ô paroles }
$$

et ces propositions, plus générales, d'Isou :

17. G. Apollinaire, «La Victoire» [1917], repris dans Calligrammes (je souligne).

18. Voir P. Albert Castanet, Tout est bruit pour qui a peur, Michel de Maule, 1999, p. 36-37.

19. "Ô paroles» suivant immédiatement les deux vers consacrés aux bruits corporels non buccaux, souligne assez clairement le devenir-langue ou devenir-lettres desdits «bruits», qui en fait autant d'éléments poétiques potentiels. 
Pourquoi le langage buccal seul se considèrerait-il poétique au détriment de tout autre moyen de communication humaine?

Les émoluments du corps entier ne devraient-ils pas être classés dans la catégorie des signes autant qu'ils répondent à la nécessité du bruit?

[...] Lintroduction de deux "instruments" inédits dans la Lettrie (les mains et les pieds s'ajoutant à la bouche) rompt la solitude du Lecteur. S'accompagnant lui-même il est plusieurs [...].

Le lecteur se démène corps et bouche dans un ordre rigoureusement inscrit par l'emploi intégral offert à chacun de ses membres.

[...] La Lettrie, bruit humain (et non simplement voix) réduit à des lettres, ouvre des sources insoupçonnées à cet art ${ }^{20}$.

Une poésie bruyante est donc (quelles qu'en soient les caractéristiques particulières) celle qui met à mal l'exigence toujours reformulée, et lénifiante, d'euphonie et d'harmonie poético-musicales : "sonorités, musique des vers», etc.; ainsi que l'impératif de clarté et d'intelligibilité du filé/nappé communicationnel toujours dominant, et aliénant : qu'il soit d'un type médiatiquement correct, ou littérairement élaboré.

«Musique» contre «musique»...

\section{Que n'allons-nous droit au phonographe?}

C'est ici qu'il faut revenir au célèbre diagnostic du Maître de la rue de Rome :

Nous assistons, en ce moment [...], à un spectacle vraiment extraordinaire, unique, dans toute l'histoire de la poésie : chaque poète allant, dans son coin, jouer sur une flûte, bien à lui, les airs qu'il lui plât; pour la première fois, depuis le commencement, les poètes ne chantent plus au lutrin ${ }^{21}$.

Dispersion des écritures - diversification des "chants», des «airs", des dictions -, aventureuse sortie hors du grand moule unique, pour laquelle, hardiment, il propose une interprétation en termes de rupture dans l'histoire de la société occidentale, à savoir :

que, dans une société sans stabilité, sans unité, il ne peut se créer d'art stable, d'art définitif. De cette organisation sociale inachevée, qui explique en même temps l'inquiétude des esprits, naît l'inexpliqué besoin d'individualité dont les manifestations littéraires présentes sont le reflet direct. (Ibid.)

20. Précisions sur ma poésie et moi [1950], Exils, 2003, p. 2I-23. C’est, précisément, sur le statut de ces «bruits» que devait se faire la rupture qui mena à l'invention des crirythmes et à l'ultra-lettrisme : pour Isou et Lemaitre, ils relevaient encore du signe et pouvaient donc faire l'objet d'une notation écrite; pour Dufrêne, comme avant lui pour Wolman, ce n'était plus le cas.

2I. Réponse à Jules Huret, Enquête sur l'Évolution littéraire [I891], Corti, I999, p. I00-IOI. 
Brandir l'équation poème = voix (qui est "chant", c'est-à-dire musique), avec pour argument ultime l'originaire oralité de toute poésie, c'est confondre la petite voix individuée (la «flûte») avec le grand Mètre anonyme («le lutrin, les grandes orgues») qui n'a de cesse de la refouler ou, du moins, de la canaliser - de la contenir : c'est contribuer à en perpétuer, envers et contre toutes les brèches fraîchement ouvertes ("pour la première fois, depuis le commencement»), l'incurable refoulement.

De cette confusion, du caractère analogique des terminologies qui s'y rapportent, rendait assez bien compte cette observation d'Édouard Dujardin :

La musique s'est révélée à Mallarmé et aux symbolistes, non point comme un art de virtuosité, concerto piano ou violon, gammes et acrobaties, mais comme la voix profonde des choses ${ }^{22}$.

Et en effet, quand Mallarmé parle de "musiques mentales», on se doute bien que, pas plus que d'instruments - ceux de «la musique entendue au concert»-, ce n'est de bouche qu'il est question, mais de «l'esprit, qui n'a que faire de rien outre la musicalité de tout».

À l'intention d'Edmund Gosse, il précisait :

Je fais de la Musique, et appelle ainsi non celle qu'on peut tirer du rapprochement euphonique des mots, cette première condition va de soi; mais l'au-delà magiquement produit par certaines dispositions de la parole, où celle-ci ne reste qu'à l'état de moyen de communication matérielle avec le lecteur comme les touches du piano. Vraiment entre les lignes et au-dessus du regard cela se passe, en toute pureté, sans l'entremise de cordes à boyaux et de pistons comme à l'orchestre, qui est déjà industriel; mais c'est la même chose que l'orchestre, sauf que littérairement ou silencieusement ${ }^{23}$.

C'est que "voix, musique, chant» doivent alors s'entendre analogiquement, comme le voulait saint Augustin, pour désigner ce quelque chose que l'on ne sait autrement désigner - ce quelque chose d'autre que l'on ne sait, que l'on ne saurait en aucune manière désigner.

Ce quelque chose d'autre, dont les poètes symbolistes (parmi lesquels Dujardin) feraient l'objet même de leur quête - et que suggérait subtilement Gautier lorsqu'il écrivait, avec une intuition merveilleuse, au chapitre xIII de Spirite (1866) :

Spirite, avec une intuition merveilleuse, rendait l'au-delà des mots, le non-sorti du verbe humain, ce qui reste d'inédit dans la phrase la mieux faite, le mystérieux, l'intime et le profond des choses, la secrète aspiration qu'on s'avoue à peine à soimême, l'indicible et l'inexprimable, le desideratum de la pensée au bout de ses efforts, et tout le flottant, le flou, le suave qui déborde du contour trop sec de la parole.

22. «De Stéphane Mallarmé au poète Ezéchiel» [1922], Mallarmé par un des siens, Messein, I936, p. 39 .

23. Lettre du io janvier 1893 . 
Mais, si la musique entendue au concert pouvait, de cette "musique»-là, de ce "non-sorti du verbe humain", donner quelque idée, - si même, il était question que la poésie lui reprît son bien, - quelle voix entendue à quel concert, ou sur quels tréteaux, pourrait aujourd'hui comme hier, donner idée de cette "voix" si indéfinissable, si insaisissable, - à quelles voix le poème, jadis comme naguère, pourrait-il, devrait-il reprendre son bien?

La voix du poète lisant ne participe concrètement du poème, que depuis le phonographe ${ }^{24}$; et, le plus souvent, de manière illustrative ou documentaire. C'est alors la voix triviale, ordinaire - ritualisée («territorialisée») : celle qui récite ou déclame, raisonne et arraisonne. L'universelle rapporteuse...

Est-ce cela que Mallarmé - trop assujetti encore aux ornières de la diction, aimablement socialisée, des salons et banquets - regrettait de n'avoir pu entendre, lorsqu'évoquant son unique rencontre avec Rimbaud, au Dîner des Vilains Bonshommes, il s'attarde sur les mains du jeune prodige de Charleville, qui aurait quelques semaines plus tôt séduit la même assemblée (ou presque) en lisant - en "récita[nt]»? - plusieurs poèmes de sa composition? Il ajoute en effet :

J'appris qu'elles avaient autographié de beaux vers; non publiés : la bouche, au pli boudeur et narquois n'en récita aucun ${ }^{25}$.

Sale caractère? Sans doute. Mais surtout, symptôme, que ce refus obstiné, et sans appel, où il faudra bien reconnaître un jour le seul authentique silence de Rimbaud...

C'est que, pour l'auteur du "Bateau ivre», qui avait déjà largement entamé son travail de sape des formes et des conceptions poétiques héritées d'une multiséculaire tradition, il fallait de toute urgence, et pour les mêmes raisons, en finir avec ce qu'il y avait de faux, d'arrogant et de dérisoire, dans la manière dont on pouvait alors dire - et écrire - les vers (la poésie).

En finir, donc, avec la lecture à voix haute - récitante - telle, du moins, qu'on la pratiquait alors: des banquets de Mallarmé à ceux des Vilains Bonshommes. Car elle était précisément ce qui empêche d'entendre ce quill y a à entendre dans le poème : "l'au-delà des mots, le non-sorti du verbe humain, ce qui reste d'inédit dans la phrase la mieux faite», soit : la voix autre - récalcitrante -, la voix de "je» qui "est un autre», la voix de l'autre. La voix comme autre ("déterritorialisée / déterritorialisante») : la seule, suivant les symbolistes, à même de restituer «tout le flottant, le flou, le suave qui déborde du

24. Inventé, simultanément, par Thomas Edison et Charles Cros, en I877-I878. 25. "Arthur Rimbaud», Divagations [1896], Gallimard, coll. «Poésies», I976. 
contour trop sec de la parole», parce qu'elle entretient de secrètes affinités vibratoires avec «la voix profonde des choses»...

Décidément, non, la voix du poème ne pouvait plus coïncider tranquillement, comme par nature, avec la voix (la "petite musique») du poète lisant - singer (signer) la voix de son Maître : «tant d'égoïstes se proclament auteurs".

$\mathrm{Ni}$, à plus forte raison, la voix de son Mètre (celle qui chantait «au lutrin»). On se souvient d'un autre épisode ${ }^{26}$ où Rimbaud aurait scandé, d'un «Merde!» retentissant, la «récitation» que faisait tel poète habitué du lieu, et ami du photographe Carjat : l'incident dégénéra, le trublion s'étant saisi de la canne-épée de Verlaine...

Il fallait une bonne fois pour toutes dissocier, dans la dissonance, l'authentique voix du sujet (ce "je» qui "est un autre») de ses clones mystificateurs, à commencer par celle de l'individu biographique et social (ce "Moi», ce «Poëte» qui n’en sont que risibles ou odieuses identifications narcissiques).

Or depuis que, par la grâce du phonographe, non seulement des poètes ont pu enregistrer des versions orales de leurs textes écrits («Le Pont Mirabeau»), mais que, grâce à l'appropriation du magnétophone par les poètes, des enregistrements ont acquis le statut d'œuvre poétique en tant que telle ("crirythmes, poèmes-partitions, audio-poèmes»; et, par anticipation ou rétroactivement selon le point de vue : Ursonate) - c'est-à-dire, depuis qu'il existe une poésie sonore -, s'impose progressivement le principe de l'intégration, au texte poétique lui-même, de la diction et de la voix propres du poète, dans leurs dimensions et implications pulsionnelles, acoustiques, pragmatiques, etc.

Ainsi, l'annonçait Tzara, à propos du "poème mouvementiste» où, au problème de la «diction», s'ajoute celui de l'"action»:

Jusqu'à maintenant on a récité les poèmes en haussant la voix et les bras. La relation entre ces deux éléments d'accentuation constituant l'intelligence artistique. Nous déclarons que les poèmes que nous écrivons maintenant ne s'adaptent plus à cette manière conventionnelle de réciter. L'acteur doit ajouter à la voix les mouvements primitifs et les bruits, de sorte que l'expression extérieure s'adapte au sens de la poésie. L'artiste a la liberté d'arranger et de composer les mouvements et les bruits d'après sa manière personnelle de comprendre le poème ${ }^{27}$.

26. À vrai dire, les nombreux témoignages qui devraient nous permettre d'en reconstituer le déroulement diffèrent tellement entre eux que l'incident lui-même finit par s'y diluer : voir J.-J. Lefrère, Arthur Rimbaud, Fayard, 200I, p. 396-40I.

27. «Le poème bruitiste», I4 juillet I9I6. 


\section{Du bruit dans le Verbe...}

Briser le cadre contraignant - mais protecteur - de la langue des vers, comme le fit brutalement Rimbaud, briser le cadre contraignant - mais protecteur - de la langue tout court, comme il commença à le faire et comme le fit, convulsivement, Artaud, comme le firent Kroutchonykh, Khlebnikov, Hausmann, Schwitters, Bryen ${ }^{28}$, futuristes et lettristes, comme l'ont fait et le feront "d'autres horribles travailleurs", c'est en quelque sorte ouvrir les vannes, c'est accueillir sans réserves à même le discours, désacralisé sans appel, cet enchantement labile des primes lalies, ces déchaînements de labiales et de dentales, ces souffles, sifflements, chuintements, cliquetis et claquements de langue, de luette, ces pétarades et glissandi, ces infantiles bouillonnements de la caverne phonatoire, ces brouillons d'actes locutoires, qui apparaissent comme autant de parasites, et suscitent un brouillage sans précédent de la chaîne parlée : "Trouver une langue» qui permette enfin que s'entende, dans le poème, ce que la langue des vers depuis toujours avait eu pour effet, sinon pour fonction, de taire, de tarir. (C'est, non moins, ce que réclamait, d'une exigence lyrique assurée de sa légitimité, l'Apollinaire de «La Victoire».)

Soit :

À la fin du $\mathrm{xx}^{\mathrm{e}}$ siècle, quand le capitalisme s'étend et se renforce, il est devenu impossible pour un artiste de continuer à faire comme si les expressions qu'il produit avaient une valeur de participation et de réconciliation affectives. De fait elles ont ou n'ont pas une valeur d'échange, et si elles jouent un rôle de réconciliation, c'est qu'elles fonctionnent comme moment idéologique dans le circuit du capital : circulation d'affects et de représentants pulsionnels couvrant une autre circulation, celle du capital [...]. Les traces de l'inconscient, les marques du désir sur les œuvres du processus secondaire sont à acheter et à vendre. Le désir est ou sera capté dans le réseau du capital, c’est-à-dire dans le processus secondaire hyperrationnel et hyperopératoire ${ }^{29}$.

... Et l'on concevra aisément le lien, qu'établirent aussi bien Artaud - voire Ponge - que Rimbaud (mais Rimbaud, le premier et avec quelle lucidité et persévérance) entre un travail poétique conçu comme une destruction émancipatrice de l'ensemble des marques et contraintes formelles relevant de la Loi du Verbe, et une volonté subversive visant, au-delà de toutes les figures et de tous les dispositifs d'autorité qui en sont les manifestations à un moment et dans une société donnés, à renverser la Loi de Dieu : justification ultime (de celle)

28. Qui, dans «L'heure du Biniou», n’hésite pas à évoquer les «mots inventés dans l'extase de sainte Hildegarde»...

29. J.-Fr. Lyotard et D. Avron, "A few words to sing" Sequenza III», dans J.-Fr. Lyotard, Dérive à partir de Marx et Freud, Union générale d'éditions, coll. "Io/I8», I973, p. 255. 
du Capital. Confirmant ainsi, a posteriori et a contrario, la nature foncièrement théologique, et aliénante, du langage poétique hérité - à laquelle (au-delà d'un progressisme de circonstance) souscrivait profondément Hugo, qui lui donna en quelque sorte in extremis sa formulation définitive (et sa caution) :

Car le mot, c'est le Verbe, et le Verbe, c'est Dieu.

J'ai souligné ailleurs ${ }^{30}$ la vision globalement rousseauiste du langage et de la poésie, commune à des poètes aussi divers que Rimbaud, René Ghil, Hugo Ball - et que résumait, à sa manière toute de désinvolture sceptique et lapidaire, Paulhan ${ }^{31}$ :

Un [...] courant secret de la littérature - secret, mais d'où sortent les œuvres les plus vivaces que l'on ait vues de nos jours - exige du poète, par quelque alchimie, une autre syntaxe, une grammaire nouvelle et jusqu’à des mots inédits, où revivrait l'innocence primitive, et je ne sais quelle adhésion perdue du langage aux choses du monde. Tel fut le rêve, et parfois la réussite, de Rimbaud, d'Apollinaire, de Joyce. Une école moderne, non pas négligeable, ${ }^{*}$ donne à l'écrivain pour premier devoir de "dissocier la matière des phrases»; une autre école l'invite à dissocier la matière des mots. ${ }^{* *}$

"Certes, admettait André Spire, il y aurait folie à nier les analogies entre la musique et "sa sœur articulée" la poésie.» Car,

$[\ldots]$ toutes deux issues du même complexe dynamique primitif : geste, cri plus ou moins modulé, parole plus ou moins articulée, par lequel l'organisme humain répondait aux excitations d'origine extérieure ou intérieure, elles furent plus tard et longtemps confondues dans une activité indivise : musique - poésie - danse, qui apparaît d'abord dans des cérémonies magiques. Ce groupe peu à peu se dissocia en combinaisons binaires : musique-parole (le chant), musique-geste (la danse, la pantomime), paroles-gestes (le théâtre), d'où se détacha, par élimination progressive de tous les autres éléments, une musique sans contenu apparent de paroles ou de gestes, la musique prétendue pure, et qu'il me paraît préférable, avec Lionel Landry, d'appeler musique autonome ${ }^{2}$.

À quoi il conviendrait d'ajouter : «une parole sans contenu apparent de musique ou de geste, la poésie au sens où on l'entend communément, et qu'il me paraît préférable, avec Bernard Heidsieck, d'appeler "poésie écrite" ou, mieux encore, poésie muette». Dès lors, toute poésie - qu'elle le fasse discrètement ou tumultueusement, visant à l'harmonie ou à la discordance, qu'elle aille, audacieusement, jusqu'au bout du processus, ou qu'elle s'arrête,

30. "R. Ghil : une mystique...», op. cit., p. 26-32.

3I. Les fleurs de Tarbes, Gallimard, coll. "Idées", I94I, p. 32. Notes de l'auteur : * C'est l'école des Mots en Liberté, dont le chef fut Marinetti. "** "Lécrivain a le droit d'employer des mots qu'il fabrique lui-même... L'écrivain exprime, il ne communique pas.» (Manifeste de Transition, signé par Georges Pelorson, Eugène Jolas, Camille Schuwer, etc.)

32. Op. cit., p. 7-8. 
prudemment, en chemin - ne saurait avoir pour objet, et pour justification ultime, que "cette remontée du langage articulé vers le frémissement communicatif des mimiques corporelles et vocales primitives» qui, d'ailleurs, ne lui est pas propre : elle n'est, en définitive, que l'effet, sur notre langage comme sur notre comportement, d'une "émotion intense», capable de renverser «des siècles de politesse sociale ou mondaine». Mais, ne faudrait-il pas pour cela $\mathrm{a}^{33}$ "enlever "du langage tout ce qui est dans le dictionnaire ou la grammaire, de la musique tout ce qui est dans le solfège" "?

Ainsi, Hugo Ball :

On voit alors parfaitement bien comment se produit le langage articulé. Je laisse galipetter les voyelles, je laisse tout simplement tomber les sons, à peu près comme miaule un chat... Des mots surgissent, des épaules de mots, des jambes, des bras, des mains de mots. Au, oi, u. Il ne faut pas laisser venir trop de mots. Un vers, c'est l'occasion de se défaire de toute la saletée ${ }^{34}$.

Et, certes, il en est peu, pour se résoudre à pareilles extrémités! Ainsi, Lyotard/Avron :

Ce dont la pseudo-langue qu'offre Visage [«Karawane», l'Ursonate ou l'instrumentation-Verbale...] est le plus proche, c'est cette langue supposée d'origine que Rousseau imaginait dans l'Essai sur l'origine des langues, et qu'il opposait au langage de communication comme la musique s'oppose à la peinture $[\ldots]:$ : la plupart des mots radicaux seraient des sons imitatifs, ou de l'accent des passions, ou de l'effet des objets sensibles : l'onomatopée s'y ferait sentir continuellement."

La fonction du simulacre est dès lors évidente : nous donner la musique qui est supposée au commencement de la parole, la musique des passions que le langage de communication refoule, mais de laquelle selon Rousseau (et peut-être Berio) il naît, dans laquelle en conséquence il doit s'annoncer et ne peut s'annoncer que comme métaphore de lui-même. Ordre figural maquillé, présenté en ordre discursif 35 .

À un tel «maquillage», à cette condition sine qua non d'un «simulacre» de langue ou de discours, accueillant à ce qui l'ébranle, pourraient bien échapper - et opposer un inentamable défi (dérision ou déni) - des œuvres basées sur un (mal)traitement particulièrement extrême du matériau vocal de la langue ou de la profération même, ou du matériau musical, comme les Crirythmes de Dufrêne, maints Audiopoèmes de Chopin, telle ou telle pièce Fluxus qui, exécutée en "concert", a pu paraitre - et paraît toujours quelquefois - étrangère (voire hostile) à la moindre organisation sonore méritant le nom de «musique»...

33. A. Spire, ibid., p. 9, citant L. Landry, op. cit., p. 158.

34. "Premier manifeste dada " (I4 juillet I9I6), trad. S. Wolff : Dada à Zurich. Le mot et l'image, Les Presses du Réel, Dijon, 2006, p. II.

35. Op. cit., p. 258-259. Les auteurs citent deux passages de l'Essai, chap. I et IV (je souligne). 
... Langue autre, langue perdue, parole imprescriptible, qu'il s'agit (que ça s'agite) de faire entendre, par les trous de la langue de l'autre, de la langue héritée, hérissée de prescriptions et de proscriptions. Ou: "mots-musique d'une langue-musique», pour reprendre la formule ghilienne, et même : lettresmusique, bruit-musique... Langue, enfin, de la poésie: de la "vraie poésie» - telle que la pressentait Laforgue la reconnaissant, naissante, dans les vers de Sagesse, à :

[...] des vagissements, des balbutiements dans une langue inconsciente ayant tout juste le souci de rimer $^{36}$.

36. Lettre à Gustave Kahn du 28 ou 29 novembre i883. Tous deux, soulignons-le, ex-assidus parmi le public des Hydropathes... 
